\title{
The relationship between root exudation, accumulation of heavy metals and symbiotrophy in peas (Pisum sativum L.)
}

\author{
Belimov A.A.*, Shaposhnikov A.I., Syrova D.S., Guro P.V., Yuzikhin O.S., \\ Azarova T.S., Sazanova A.L., Sekste E.A., Safronova V.I. \\ All-Russia Research Institute for Agricultural Microbiology, St. Petersburg, Russia \\ *email: belimov@rambler.ru
}

After screening of 120 pea genotypes from the N.I. Vavilov Institute of Plant Genetic Resources, ten genotypes contrasting in the accumulation of heavy metals (HMs) such as $\mathrm{Cd}, \mathrm{Co}, \mathrm{Cr}, \mathrm{Cu}, \mathrm{Ni}, \mathrm{Pb}, \mathrm{Sr}$ and $\mathrm{Zn}$ from soil were selected. Inoculation with symbiotic microorganisms reduced HMs contents in these plants. Genotypic differences were revealed in the composition of root exudates (primary and aromatic organic acids, glycosides and amino acids), rhizosphere microbial communities, and mobile forms of HMs or nutrients in the rhizosphere. When dividing pea genotypes into two separate groups with low and high HM content, the components of root exudates determining the differences between them were identified. The highest values of exudation were found for the low-accumulating group. Significant correlations between $\mathrm{HMs}(\mathrm{Ni}, \mathrm{Pb}$, and $\mathrm{Zn})$ content in pea shoots and the intensity of root exudation of some organic compounds were found. However, it was not possible to group the studied genotypes according to the exudation activity into separate clusters. Also, no correlations were found between the intensity of root exudation and the concentration of mobile forms of HMs in the rhizosphere. The results showed that some components of root exudates may play a role in the characterization of plant genotypes in terms of their ability to accumulate HMs. For example, organic acids can chelate metal ions, making them less available for assimilation by the roots. Glycosides can affect the biological activity of rhizosphere processes. Symbiotic parameters (the number and biomass of nodules, nitrogen fixation, formation of mycorrhizal structures, effect of inoculation on nutrient uptake) negatively correlated with the exudation of some components. It is likely that genotypes with low exudation retain more photosyntates for the development of a symbiotic apparatus. The results highlighted the complexity, complexity and interdependence of the observed processes and patterns.

Acknowledgements: The work was supported by the Russian Science Foundation (grant 19-16-00097). 